\title{
Identification of differentially expressed miRNAs associated with thermal injury in epidermal stem cells based on RNA-sequencing
}

\author{
HAO-TIAN RONG ${ }^{1,2}$ and DE-WU LIU ${ }^{1}$ \\ ${ }^{1}$ Burns Institute, The First Affiliated Hospital of Nanchang University; ${ }^{2}$ First Clinical Medical College, \\ Nanchang University, Nanchang, Jiangxi 330006, P.R. China
}

Received October 28, 2018; Accepted November 6, 2019

DOI: $10.3892 /$ etm. 2020.8448

\begin{abstract}
Current research indicates that epidermal stem cells (EpSCs) play an important role in promoting wound healing, but the mechanism of action of these cells during wound repair following thermal damage remains unclear. In the present study, the trypsin digestion method was used to isolate human EpSCs and the cells were incubated in a $51.5^{\circ} \mathrm{C}$ water tank for $35 \mathrm{sec}$ to construct a thermal injury model. The differentially expressed miRNAs were identified using high-throughput sequencing technology, and bioinformatic methods were used to predict their target genes and signaling pathways that may be involved in wound repair. A total of 33 miRNAs including, hsa-miR-1973, hsa-miR-4485-3p, hsa-miR-548-5p, hsa-miR-212-3p and hsa-miR-4461 were upregulated, whereas 21 miRNAs including, hsa-miR-4520-5p, hsa-miR-4661-5p, hsa-miR-191-3p, hsa-miR-129-5p, hsa-miR-147b and hsa-miR-6868-3p were downregulated following thermal injury of the human EpSCs. The bioinformatic analysis indicated that the differentially expressed miRNAs are involved in biological processes such as cell proliferation and differentiation, cell growth apoptosis, cell adhesion and migration. The results showed that there is a differential expression pattern of miRNAs after thermal injury of human EpSCs and these differences are involved in the regulation of the wound healing process. These findings provide new clues for further study of the wound healing mechanism and targeted therapy.
\end{abstract}

\section{Introduction}

Burn is a common type of traumatic disease. Heat, chemicals, currents and radiation are the common causes of burn injury. Among the numerous injury factors, heat is the most common factor, and thermal damage is always combined with burns $(1,2)$.

Correspondence to: Professor De-Wu Liu, Burns Institute, The First Affiliated Hospital of Nanchang University, 17th Yongwai Street, Donghu, Nanchang, Jiangxi 330006, P.R. China

E-mail: dewuliu@126.com

Key words: epidermal stem cells, microRNA profiling, thermal damage, high-throughput sequencing
The injury factors of thermal burns are usually directly or indirectly caused by individuals touching high-temperature solids, liquids or gases (3). Cell morphology, metabolism and function are altered in the tissues at the burn site and the level of damage is related to many factors which are associated with the ratio between the level of the heat energy and the duration. The earliest cell histological changes after injury are found in the epidermis which reveals the redistribution of chromatin in the nucleus. With further damage, the cytoplasm of basal cells and the nuclei of full-thickness cells begin to swell or even become necrotic. If the heat of injury further increases, the epidermis immediately undergoes coagulative necrosis and even carbonization, eventually causing serious damage to the local tissue and even to the entire body (4).

The skin is the largest organ, and healthy conditions of the skin can aid humans to prevent attacks from the external environment (5). Similar to other stem cells, epidermal stem cells (EpSCs), derived from skin tissue, display functions of self-proliferation and differentiation and can maintain normal epidermal structure (6). The proliferation, differentiation and apoptosis of EpSCs are coordinated by multiple gene pathways (7), thus EpSCs play an important role in promoting wound healing. Upregulation of the regeneration process of EpSCs and inhibition of their apoptotic processes can accelerate the repair of damaged wound tissue (8). EpSCs synthesize and secrete a variety of biologically active substances such as immunomodulatory factors, angiogenic factors, anti-apoptosis factors, antioxidants and cell chemokines. Research has confirmed that cross-linking between multiple signaling pathways forms a strict and orderly regulatory network, and any regulatory changes in the network may interfere with the wound healing process (8).

MicroRNAs (miRNAs) are important factors that regulate the proliferation, differentiation and apoptosis of EpSCs (9). miRNAs are small, non-coding RNAs approximately 22 nucleotides (nt) in length that are encoded by higher eukaryotic genomes. miRNAs can degrade to mRNAs and inhibit mRNA transcription and enable post-transcriptional regulation (10). Studies have shown that miRNAs play an important role in skin tissue proliferation and wound healing (5). There have been studies confirming that miRNA-203 is an epithelial tissue-specific miRNA that not only participates in skin development but also induces the differentiation of EpSCs into various specialized cells (11). In the present study, the trypsin 
digestion method was used to isolate human EpSCs. The cells were then incubated in a $51.5^{\circ} \mathrm{C}$ water tank for $35 \mathrm{sec}$ to construct a thermal injury model. The differentially expressed miRNAs were identified using high-throughput sequencing technology, and bioinformatic methods were used to predict their target genes and signaling pathways that may be involved in wound repair.

\section{Materials and methods}

Sample collection. Normal skin samples were obtained from 10 male patients, median age 25 years (range 18-35 years) who underwent autologous grafting with epidermis at the Burn Center, The First Affiliated Hospital of Nanchang University from January 2016 to June 2016. The present study was conducted according to The Declaration of Helsinki and was approved by the Ethics Committee of Nanchang University. All patients provided written informed consent prior to the study start.

Epidermal stem cell isolation and culture. Following simple treatment, the specimens were repeatedly rinsed three times with $1 \%$ penicillin-streptomycin in phosphate-buffered saline (PBS), added to a concentration of $0.25 \%$ trypsin $+0.02 \%$ ethylene diamine tetraacetic acid (EDTA), and allowed to stand at $4^{\circ} \mathrm{C}$ for $8-10 \mathrm{~h}$. The epidermal and dermal layers of the epidermis were separated, and the separated epidermal layers were crushed into tissue homogenates and subsequently $2 \mathrm{ml}$ of $0.25 \%$ trypsin $+0.02 \%$ EDTA were added and digestion was carried out at $37^{\circ} \mathrm{C}$ for $10 \mathrm{~min}$. Defined K-SFM ( $2 \mathrm{ml}$ ) containing $10 \%$ fetal bovine serum was added to stop the trypsin digestion, followed by 200 mesh sieve filtration. The filtrate was collected in a $15-\mathrm{ml}$ centrifuge tube and centrifuged at $110 \mathrm{x} \mathrm{g}$ for $5 \mathrm{~min}$ at $37^{\circ} \mathrm{C}$. The supernatant was discarded. A total of $5 \mathrm{ml}$ of $1 \%$ penicillin-streptomycin in PBS was added, followed by centrifugation at $110 \mathrm{x} \mathrm{g}$ for $5 \mathrm{~min}$ at $37^{\circ} \mathrm{C}$ and the supernatant was removed. Following centrifugation, $10 \mathrm{ml}$ of Defined K-SFM (Gibco; Thermo Fisher Scientific, Inc.) was added to the cell layer to resuspend the cells, and after the count, the concentration was adjusted to $3 \times 10^{6}$ cells $/ \mathrm{ml}$. Subsequently, the cells were inoculated in a pre-plated type IV collagen culture dish, and the culture dish was placed at $37^{\circ} \mathrm{C}$. Following incubation for $20 \mathrm{~min}$ in a $\mathrm{CO}_{2}$ incubator, the upper unattached cell suspension was aspirated and washed twice with PBS, and $5 \mathrm{ml}$ of the culture medium was added to the culture dish, which was then labeled and placed in an incubator. Subsequently, IV collagen was used to prepare for adherence screening, and the positive expression of ck19 and integrin $\beta 1$ was confirmed by immunochemical staining $(12,13)$.

Human epidermal stem cell heat injury treatment. The EpSCs were cultured at $5^{\circ} \mathrm{C}$ in a $5 \% \mathrm{CO}_{2}$ saturated humidity incubator and the culture fluid was changed every other day. When the cell density reached $90 \%$ and most of the cells grew well, the culture fluid was changed again. A thermostatic water tank was preheated for $30 \mathrm{~min}$ in advance; the temperature was set to $51.5^{\circ} \mathrm{C}$, sealing material and timer were prepared, and a Petri dish was sealed with sealing material. The cells were randomly divided into two groups, control group (group 1) and experimental group (group 2). For the experimental group, the sealed Petri dish was suspended in a $51.5^{\circ} \mathrm{C}$ water tank and incubated for $35 \mathrm{sec}$. The timer was set. The control dish was placed in a $37^{\circ} \mathrm{C}$ water tank and also incubated for $35 \mathrm{sec}$. The procedure was the same as above and as previously described (14). The treated cells were observed under a fluorescent inverted phase contrast microscope (magnification, x100) to detect cell morphology and the number of apoptotic cells. The samples were further collected after $6 \mathrm{~h}$ of culture to extract RNA.

Total RNA extraction and purification. Total RNA was isolated using Magzol reagent solution (Thermo Fisher Scientific, Inc.) and purified using the RNeasy Mini kit (Qiagen $\mathrm{GmbH}$ ), according to the manufacturer's protocol. RNA quality and quantity were measured using a NanoDrop Spectrophotometer (ND-1000; NanoDrop; Thermo Fisher Scientific, Inc.). RNA integrity was determined by electrophoresis on a denatured agarose gel prepared in house. On the denaturing gels, the $28 \mathrm{~S}$ and $18 \mathrm{~S}$ ribosomal RNA bands were visible, indicating that the extracted total RNA was intact, the RNA degradation and contamination were low, and the extracted total RNA showed high purity levels. The A260/A280 ratio is a measure of RNA purity. A ratio of $<1.8$ indicates sample contamination and a ratio of $>2.0$ indicates RNA hydrolysis, between 1.8 and 2.1 , indicating that the RNA purity meets high-throughput sequencing requirements.

High-throughput sequencing technology library construction and quality inspection. Small RNA high-throughput sequencing was performed by Guangzhou RiboBio Co., Ltd., and the library was constructed and sequenced as follows: $1 \mu \mathrm{g}$ of total RNA ( $\geq 50 \mathrm{ng}$ small RNA) was used as the initial amount of RNA sample. The sample was supplemented with water to make the total reaction volume $7 \mu$ l. Subsequently, the library was constructed using a small RNA sample preparation kit (New England BioLabs, Inc.), according to the manufacturer's protocol. After the library was constructed, the concentration of the sample was determined by Qubit 2.0 (Thermo Fisher Scientific, Inc.), and the sample in the library was diluted to a concentration of $1 \mathrm{ng} / \mu \mathrm{l}$. Library quality tests were performed using an Agilent 2200 TapeStation (Agilent Technologies, Inc.); cDNA fragments with an insert size of 18-40 nt were obtained by gel electrophoresis.

Data analysis. We annotated clean sequences, as well as analyzed the composition and expression difference of all types of miRNAs. Bioinformatic methods were used for family analysis of the differentially expressed miRNAs, and Target-Scan (www.targetscan.org), miRDB (www.mirdb.org), miRanda software (www.microrna.org) and high-throughput CLIP-seq data (www.clipdb.ncrnalab.org) were used to predict miRNA target genes, and miRNAs that were significantly differentially expressed in this experiment were analyzed. Target gene prediction was performed, and then the predicted target genes were analyzed by Kyoto Encyclopedia of Genes and Genomes (KEGG) (www.genome.jp/kegg) biological pathway enrichment analysis and Gene Ontology (GO) (www.geneontology.org) gene function enrichment analysis. Statistical analysis was performed using SPSS software (version 22.0; IBM Corp.). 
$G O$ analysis. The gene list of the differentially expressed upregulated and downregulated genes was prepared, and the data were imported into the GO database, human species was selected, and then calculation was carried out. The P-value related to the enrichment of GO terms was calculated by the default statistical algorithm of the GO analysis database. The smaller the P-value, the more notable the entry of the GO term, and a term entry with $\mathrm{P} \leq 0.05$ was considered to be statistically significant. The base 10 logarithm of the P-value was converted to a negative value, and an enrichment score was obtained. The enrichment score value represents the possibility that the differentially expressed mRNA is enriched in the term entry. The higher the enrichment score more notable the entry. Enrichment for significant features was sorted in descending order, and the GO Enrichment histogram was plotted in Microsoft Excel.

Pathway analysis. The data were imported into the KEGG database and human species data were selected and investigations were performed. The significance score of differential gene enrichment for each pathway was calculated via the hypothesis testing to obtain significant P-values. The smaller the P-value the more notable the respective biological pathway. A biological pathway with $\mathrm{P}<0.05$ was considered to be statistically significant. The base 10 logarithm of the P-value was converted to a base-negative logarithmic scale, and the enrichment score value represents the possibility that the differentially expressed mRNA is enriched in this biological pathway. The higher the enrichment score the more important this pathway was. Specific signal paths and data can be exported in the system. P-values for significant pathways were ranked in ascending order, and the Log p histogram of the KEGG pathways were plotted by Microsoft Excel.

\section{Results}

Changes in epidermal stem cell growth after heat injury. As a result of observation under an inverted microscope, the EpSCs were found to be firmly attached, with a small and rounded shape and well refraction was noted when they were inoculated. After culturing for 2 days, the cells covered more than 90\% and the clones grew rapidly. The cells attached firmly. The control group showed no obvious reduction in the number of cells after a $37^{\circ} \mathrm{C}$ water bath. The cell shape was round, and cells were firm. After the $51.5^{\circ} \mathrm{C}$ water bath exposure in the experimental group, the number of cells was significantly decreased, the cells showed irregular shapes, and were not attached firmly. Immunochemical staining images showed that $\beta 1$ integrin and CK19 were positively expressed which are characteristic of EpSCs (Fig. 1).

Extraction and qualification of total RNA. The quality test results of the two groups of total RNA spectrophotometry met the experimental requirements, and the miRNA cluster plots for experimental and control groups are presented in Fig. 2.

Analysis of the differential expression of the miRNAs between the two groups. Table I documents the variations and patterns of miRNA expression between the two groups. We analyzed the data in the results of the high-throughput sequencing, statistical analysis of the two groups of samples for the differential expression of miRNA was performed to determine whether the difference was significant, and $\log 2$ ratio and scatter plots were used to compare the differences in miRNA expression. In Table IA, the experimental group has a numerical value, while the control group is 0 . As the negative infinity cannot visually demonstrate the different relationships, the control group takes 0.01 to calculate, and the $\log 2$ value is obtained after the correction. The formula is $\log 2$ (experimental group/control group). In Table IB, the expression level of certain miRNAs in the experimental group was 0 , indicating that the sequence of the miRNA was not detected in all samples of the group. This did not mean that there was no expression, but the expression level was low. Low expression levels are difficult to measure. Moreover, there were identical values due to the low expression level of various miRNAs. In order to accurately reflect the difference relationship, the system calculated the corrected values as equal.

Differences in miRNA expression between samples (Fig. 3) showed differences in the expression of all miRNAs, with a $\log 2$ (fold change) $\geq 1$ threshold. Red represents miRNAs with upregulated expression, gray circles indicate miRNAs with no significant difference in expression, and green circles represent miRNAs with downregulated expression. $\log 2 \geq 1$ is the upregulation threshold, and -1 is the downregulation threshold.

There were 33 significantly upregulated miRNAs and 21 significantly downregulated miRNAs. Among them, hsa-miR-1973 exhibited the most obvious upregulation and hsa-miR-4520-5p exhibited the most significant downregulation.

Prediction of differentially expressed miRNA target genes and KEGG pathway and GO analyses. Target genes were predicted for the miRNAs with significant differential expression in the present study, using Target-Scan, miRDB, miRanda and high-throughput CLIP-seq data software. The prediction results were further screened and organized, and the final results revealed a total of 391 predicted target genes.

KEGG analysis of the target genes of the miRNAs, with a $\mathrm{P}<0.05 \mathrm{KEGG}$ enrichment pathway degree as a significant threshold, resulted in significant enrichment of 32 KEGG pathways (Table II). Among them, the processes associated with wound healing include: FoxO signaling pathway regulates cell cycle and apoptosis; mTOR signaling pathway regulates cell metabolism and proliferation; ErbB signaling pathway regulates cell proliferation, differentiation and migration; insulin signaling pathway participates in the occurrence of diabetes, participates in the metabolism of three major substances and promotion of cell proliferation; focal adhesion signaling pathway participates in adhesion of cell matrix, regulates cell proliferation, differentiation, apoptosis, and migration; PI3K-Akt signaling pathway regulates transcription and translation of genes, and participates in cell biological metabolism, proliferation and apoptosis process; MAPK signaling pathway is highly conserved and involved in the regulation of cell proliferation, differentiation and migration; cell cycle regulates cell mitosis; Activation of Hippo signaling pathway leads to apoptosis, prevents cell overgrowth, and restricts organ size; Wnt signaling pathway regulates cells the final direction of growth involved in the proliferation of 
A

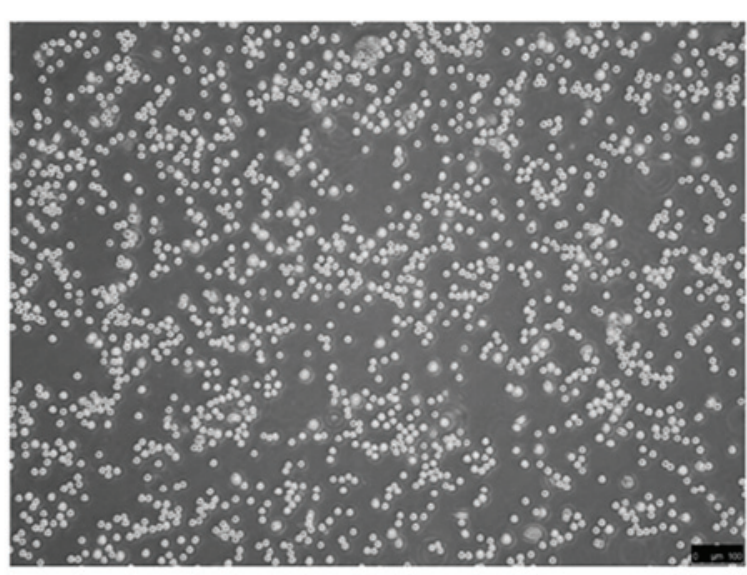

C

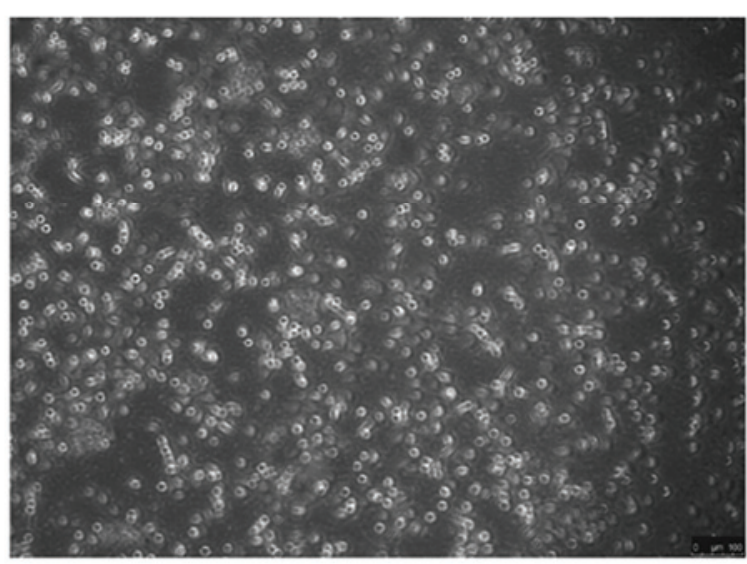

E

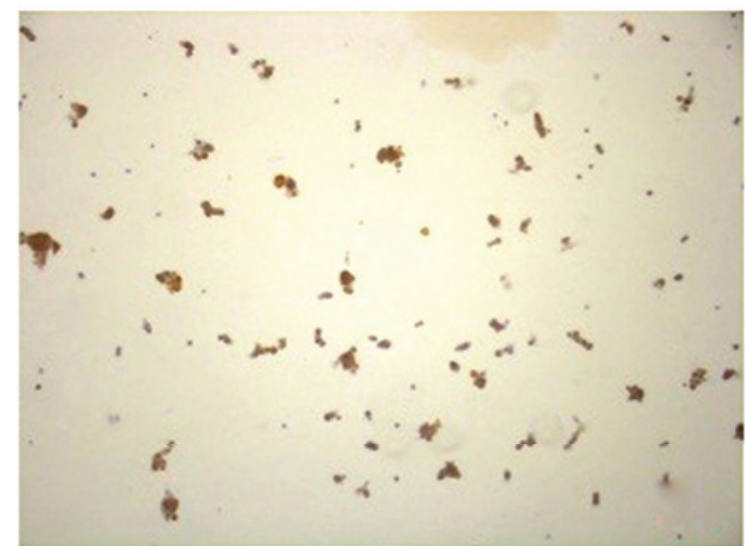

B

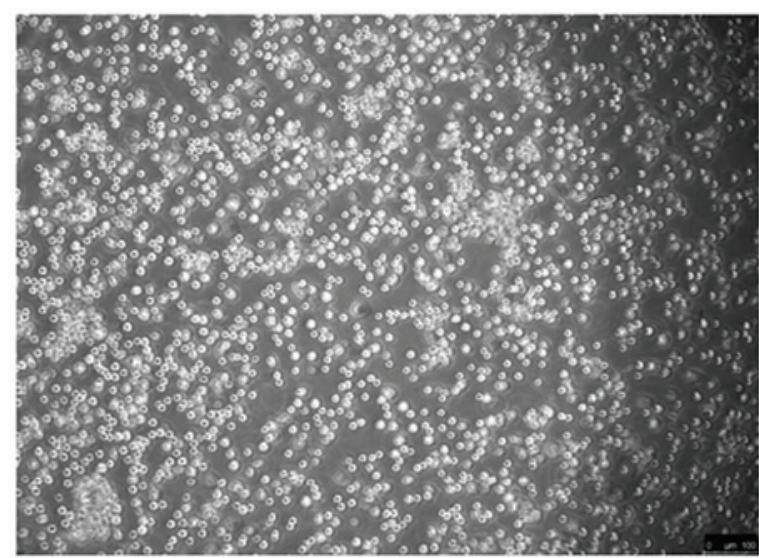

D

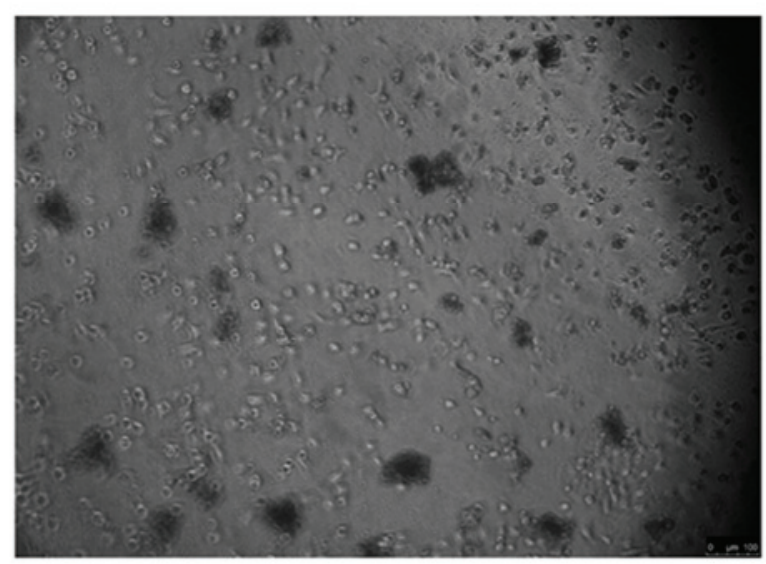

F

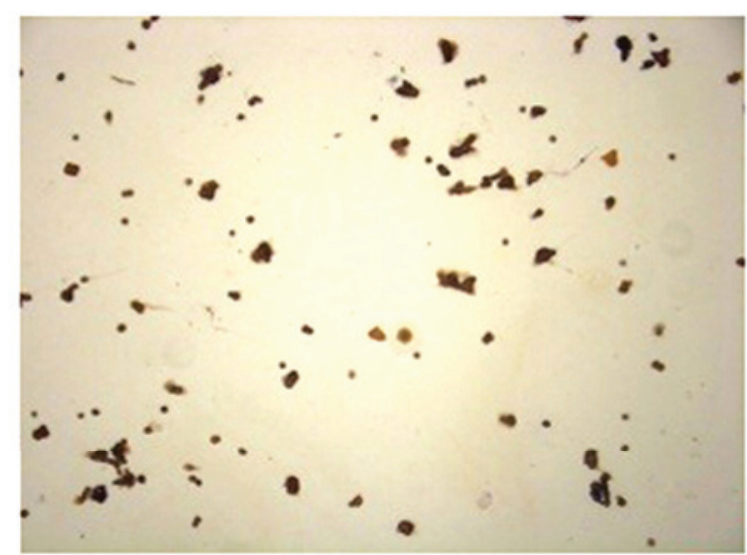

Figure 1. Morphological observation and identification of EpSCs (magnification, x100). EpSCs at (A) initial inoculation, (B) after culturing for 2 days, (C) after a $37^{\circ} \mathrm{C}$ water bath (control group) and (D) after a $51.5^{\circ} \mathrm{C}$ water bath (experimental group). EpSCs showing (E) positive expression of integrin $\beta 1$ and (F) positive expression of CK19. EpSCs, epidermal stem cells.

stem cells and metabolism process. Fig. $4 \mathrm{~A}$ is a statistical graph of the KEGG enrichment pathways in the sample.

The GO analysis of the cell composition demonstrated each part of the cell and the cell internal and external environments. GO analysis of the molecular function demonstrated the activity of the target gene product at the molecular level, such as gene transcription, translation and expression, and the binding and catalysis in metabolic processes. Furthermore, $\mathrm{GO}$ analysis of the biological processes revealed involvement of cell proliferation, differentiation, signal transduction and apoptosis processes (Fig. 4B). The above results showed that they play a role in maintaining the stability of the cell internal and external environment, gene transcription, translation and expression, biochemical metabolic processes, cell proliferation and differentiation, signal transduction, and apoptosis.

\section{Discussion}

Wound healing is a complex physiological process and is utilized to maintain the integrity of the skin. It is a complex and dynamic process, requiring the well-orchestrated cooperation of different types of cells (15). Wound healing is often characterized as four sequential but overlapping phases: Hemostasis, inflammation, proliferation and remodeling (16). 


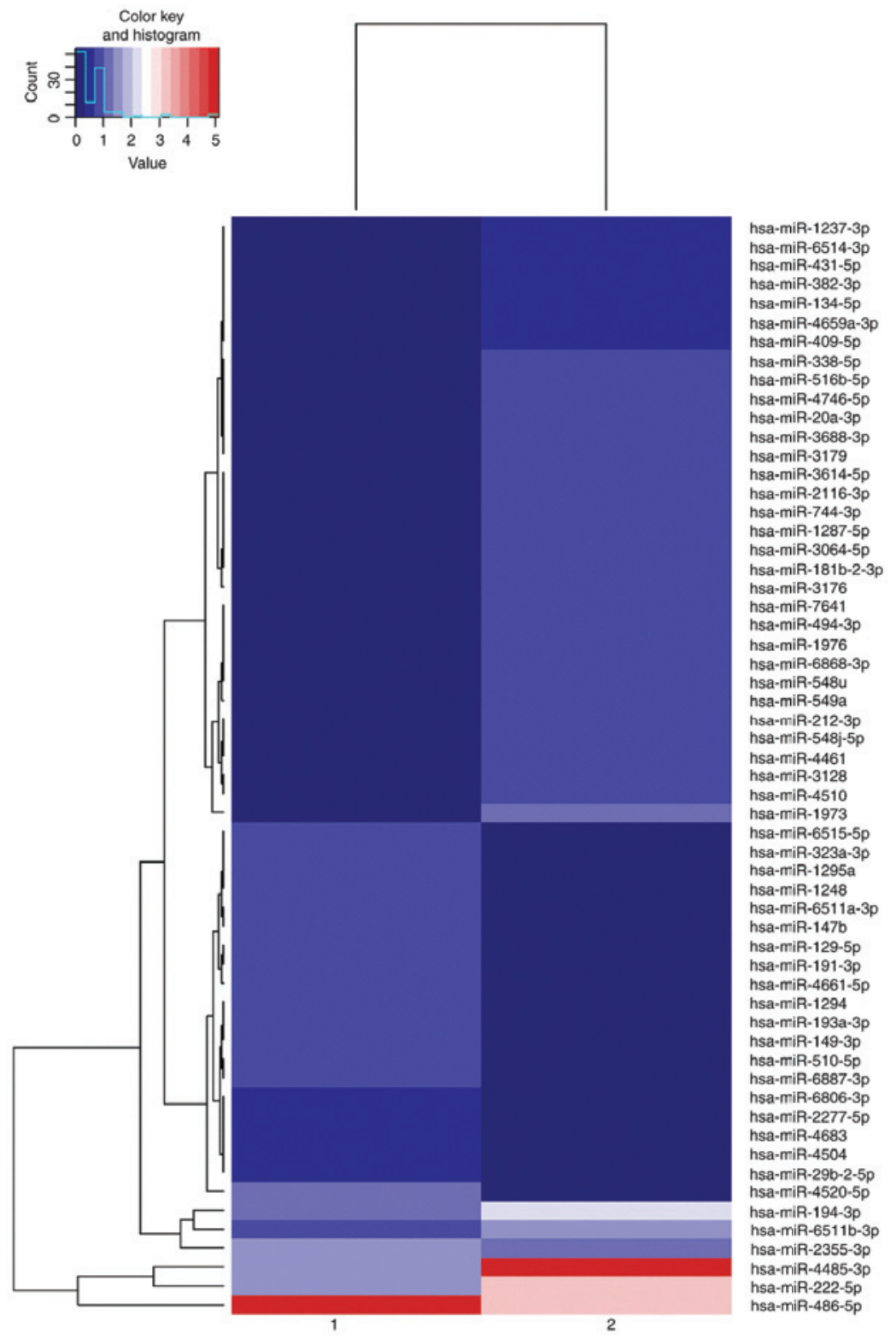

Figure 2. miRNA cluster plots for the experimental and control groups. 1 represents the control group and 2 represents the experimental group. Red represents the miRNAs that are highly expressed in the sample and blue represents the miRNAs that have low expression in the sample. miRNA, microRNA.

Current research has confirmed that miRNAs play a pivotal role in the regulation of wound repair networks (17). miRNAs are important regulators of skin wound healing, especially in the term of transition from the inflammatory to the proliferative phase (17). Recent clinical trials have demonstrated that modulation of miRNA expression by administration of specific miRNA mimics or inhibitors exhibited beneficial effects on a variety of diseases, such as cancer and viral infection (18-20).

High-throughput sequencing enables the sequencing of hundreds of thousands to millions of DNA molecules in parallel. With the advancement in research, high-throughput sequencing technology has been more widely used in the field of basic and clinical medicine, and has become an important tool for elucidating the physiological and pathological processes of the body from the molecular level (21). The present study sequenced the target genes and correlated bioinformatic analysis to provide preliminary experimental support for further research on gene-related targeted therapies.
A thermal injury model was constructed, and the results demonstrated that cell morphology was significantly altered. The total RNA of EpSCs was extracted, followed by high-throughput sequencing, and related bioinformatic analysis was performed. It was found that 33 of the differentially expressed miRNAs in the experimental group were upregulated and 21 were downregulated. hsa-miR-1973 exhibited the most significant increase in expression, whereas hsa-miR-4520-5p exhibited the most significant decrease. The significantly upregulated miRNAs also included, hsa-miR-4485-3p, hsa-miR-548j-5p, hsa-miR-212-3p and hsa-miR-4461, while the downregulated miRNAs included, hsa-miR-4661-5p, hsa-miR-191-3p, hsa-miR-129-5p, hsa-miR-147b and hsa-miR-6868-3p. Family analysis of the differentially expressed miRNAs and prediction of target genes found that they are involved in a series of signaling pathways that regulate biological processes such as cell proliferation and differentiation, growth and apoptosis, and cell migration. In terms of 
Table I. miRNAs with significantly upregulated and downregulated expression in the experimental group when compared with the control group.

A, Upregulated miRNAs

\begin{tabular}{|c|c|c|c|c|}
\hline miRNA_ID & Control group & Experimental group & $\log 2$ (fold change) & P-value \\
\hline hsa-miR-4485-3p & 1.7896 & 33.5333 & 4.2279 & $1.2 \times 10^{-7}$ \\
\hline hsa-miR-1973 & 0.0000 & 1.2268 & 6.9388 & $2.1 \times 10^{-4}$ \\
\hline hsa-miR-548j-5p & 0.0000 & 0.9931 & 6.6339 & $7.2 \times 10^{-4}$ \\
\hline hsa-miR-212-3p & 0.0000 & 0.9931 & 6.6339 & $7.2 \times 10^{-4}$ \\
\hline hsa-miR-4461 & 0.0000 & 0.9931 & 6.6339 & $7.2 \times 10^{-4}$ \\
\hline hsa-miR-4510 & 0.0000 & 0.9347 & 6.5464 & $1.0 \times 10^{-3}$ \\
\hline hsa-miR-3128 & 0.0000 & 0.9347 & 6.5464 & $1.0 \times 10^{-3}$ \\
\hline hsa-miR-549a & 0.0000 & 0.8763 & 6.4534 & $1.4 \times 10^{-3}$ \\
\hline hsa-miR-494-3p & 0.0000 & 0.8179 & 6.3539 & $1.9 \times 10^{-3}$ \\
\hline hsa-miR-7641 & 0.0000 & 0.8179 & 6.3539 & $1.9 \times 10^{-3}$ \\
\hline hsa-miR-1976 & 0.0000 & 0.8179 & 6.3539 & $1.9 \times 10^{-3}$ \\
\hline hsa-miR-6868-3p & 0.0000 & 0.8179 & 6.3539 & $1.9 \times 10^{-3}$ \\
\hline hsa-miR-548u & 0.0000 & 0.8179 & 6.3539 & $1.9 \times 10^{-3}$ \\
\hline hsa-miR-2116-3p & 0.0000 & 0.7595 & 6.247 & $2.8 \times 10^{-3}$ \\
\hline hsa-miR-3614-5p & 0.0000 & 0.7595 & 6.247 & $2.8 \times 10^{-3}$ \\
\hline hsa-miR-744-3p & 0.0000 & 0.7595 & 6.247 & $2.8 \times 10^{-3}$ \\
\hline hsa-miR-1287-5p & 0.0000 & 0.7595 & 6.247 & $2.8 \times 10^{-3}$ \\
\hline hsa-miR-3064-5p & 0.0000 & 0.7595 & 6.247 & $2.8 \times 10^{-3}$ \\
\hline hsa-miR-181b-2-3p & 0.0000 & 0.7595 & 6.247 & $2.8 \times 10^{-3}$ \\
\hline hsa-miR-3176 & 0.0000 & 0.701 & 6.1313 & $4.1 \times 10^{-3}$ \\
\hline hsa-miR-516b-5p & 0.0000 & 0.6426 & 6.0058 & $6.1 \times 10^{-3}$ \\
\hline hsa-miR-338-5p & 0.0000 & 0.6426 & 6.0058 & $6.1 \times 10^{-3}$ \\
\hline hsa-miR-4746-5p & 0.0000 & 0.6426 & 6.0058 & $6.1 \times 10^{-3}$ \\
\hline hsa-miR-20a-3p & 0.0000 & 0.6426 & 6.0058 & $6.1 \times 10^{-3}$ \\
\hline hsa-miR-3688-3p & 0.0000 & 0.6426 & 6.0058 & $6.1 \times 10^{-3}$ \\
\hline hsa-miR-3179 & 0.0000 & 0.6426 & 6.0058 & $6.1 \times 10^{-3}$ \\
\hline hsa-miR-6514-3p & 0.0000 & 0.5842 & 5.8684 & $9.2 \times 10^{-3}$ \\
\hline hsa-miR-1237-3p & 0.0000 & 0.5842 & 5.8684 & $9.2 \times 10^{-3}$ \\
\hline hsa-miR-431-5p & 0.0000 & 0.5842 & 5.8684 & $9.2 \times 10^{-3}$ \\
\hline hsa-miR-382-3p & 0.0000 & 0.5842 & 5.8684 & $9.2 \times 10^{-3}$ \\
\hline hsa-miR-134-5p & 0.0000 & 0.5842 & 5.8684 & $9.2 \times 10^{-3}$ \\
\hline hsa-miR-4659a-3p & 0.0000 & 0.5842 & 5.8684 & $9.2 \times 10^{-3}$ \\
\hline hsa-miR-409-5p & 0.0000 & 0.5842 & 5.8684 & $9.2 \times 10^{-3}$ \\
\hline
\end{tabular}

B, Downregulated miRNAs

\begin{tabular}{|c|c|c|c|c|}
\hline miRNA_ID & Control group & Experimental group & $\log 2$ (fold change) & P-value \\
\hline hsa-miR-4520-5p & 1.1185 & 0.0000 & -6.8054 & $2.9 \times 10^{-4}$ \\
\hline hsa-miR-4661-5p & 0.8948 & 0.0000 & -6.4835 & $1.0 \times 10^{-3}$ \\
\hline hsa-miR-191-3p & 0.8389 & 0.0000 & -6.3904 & $1.4 \times 10^{-3}$ \\
\hline hsa-miR-129-5p & 0.8389 & 0.0000 & -6.3904 & $1.4 \times 10^{-3}$ \\
\hline hsa-miR-147b & 0.7829 & 0.0000 & -6.2908 & $1.9 \times 10^{-3}$ \\
\hline hsa-miR-6868-3p & 0.7829 & 0.0000 & -6.2908 & $1.9 \times 10^{-3}$ \\
\hline hsa-miR-323a-3p & 0.727 & 0.0000 & -6.1839 & $2.8 \times 10^{-3}$ \\
\hline hsa-miR-6515-5p & 0.727 & 0.0000 & -6.1839 & $2.8 \times 10^{-3}$ \\
\hline hsa-miR-1295a & 0.727 & 0.0000 & -6.1839 & $2.8 \times 10^{-3}$ \\
\hline hsa-miR-1248 & 0.727 & 0.0000 & -6.1839 & $2.8 \times 10^{-3}$ \\
\hline hsa-miR-193a-3p & 0.6711 & 0.0000 & -6.0685 & $4.1 \times 10^{-3}$ \\
\hline hsa-miR-1294 & 0.6711 & 0.0000 & -6.0685 & $4.1 \times 10^{-3}$ \\
\hline
\end{tabular}


Table I. Continued.

B, Downregulated miRNAs

\begin{tabular}{|c|c|c|c|c|}
\hline miRNA_ID & Control group & Experimental group & $\log 2$ (fold change) & P-value \\
\hline hsa-miR-149-3p & 0.6711 & 0.0000 & -6.0685 & $4.1 \times 10^{-3}$ \\
\hline hsa-miR-6887-3p & 0.6152 & 0.0000 & -5.943 & $6.1 \times 10^{-3}$ \\
\hline hsa-miR-510-5p & 0.6152 & 0.0000 & -5.943 & $6.1 \times 10^{-3}$ \\
\hline hsa-miR-486-5p & 29.528 & 9.0552 & -1.7053 & $7.4 \times 10^{-3}$ \\
\hline hsa-miR-2277-5p & 0.5592 & 0.0000 & -5.8053 & $9.2 \times 10^{-3}$ \\
\hline hsa-miR-6806-3p & 0.5592 & 0.0000 & -5.8053 & $9.2 \times 10^{-3}$ \\
\hline hsa-miR-4683 & 0.5592 & 0.0000 & -5.8053 & $9.2 \times 10^{-3}$ \\
\hline hsa-miR-4504 & 0.5592 & 0.0000 & -5.8053 & $9.2 \times 10^{-3}$ \\
\hline hsa-miR-29b-2-5p & 0.5592 & 0.0000 & -5.8053 & $9.2 \times 10^{-3}$ \\
\hline
\end{tabular}

miRNA_ID column shows the differentially expressed miRNAs. Experimental group and control group columns show the miRNA expression in the groups of samples. $\log 2$ (fold change) is the base 2, logarithmic differential expression multiple. P-value column shows the significant level P-values. Due to the low expression level of some miRNAs, in order to accurately reflect the difference relationship, the system calculated the corrected values as equal, thus the P-values are the same.

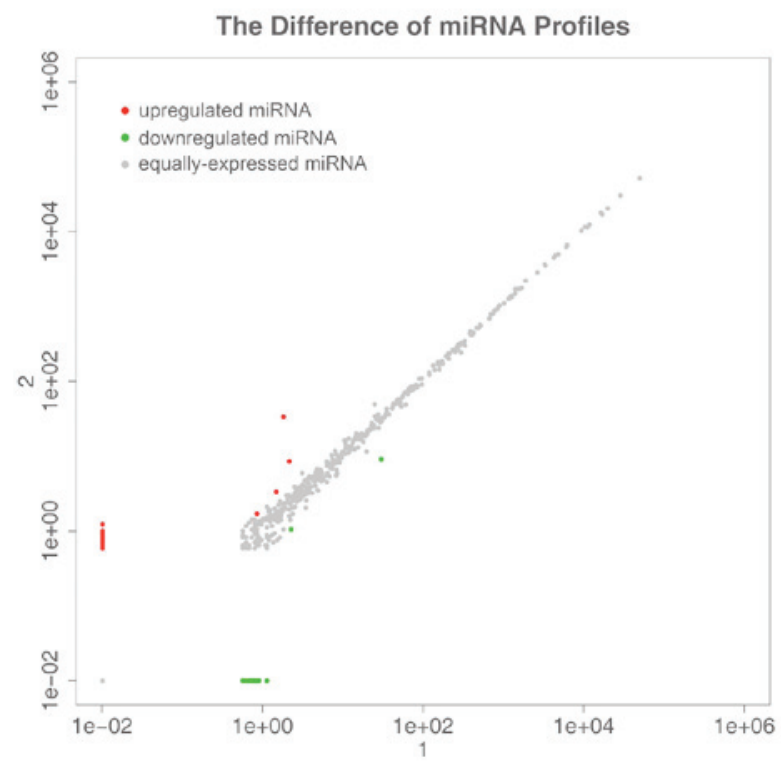

Figure 3. miRNAs with differential expression in the experimental and control samples. 1 represents the control group and 2 represents the experimental group. Red circles represent miRNAs with upregulated expression, gray circles indicate miRNAs with no significant difference and green circles represent miRNAs with downregulated expression in the two groups. miRNA, microRNA.

wound healing, miR-155 can be involved in cell migration and transformation. Overexpression of miR-155 at the wound edge can accelerate wound healing mediated by enhanced keratinocyte migration (22). It has also been reported that miRNAs are important regulators of inflammatory and tissue repair that act through translation processing of target mRNAs (23). In terms of cell proliferation, miR-1973 and miR-191-3p play an important role in breast cancer proliferation and lymph node metastasis $(24,25)$. Tissue regeneration, tissue repair and regeneration depend on the function of miRNAs, which are currently widely used in the tissue engineering of cartilage, bone and skeletal muscle (26).

GO analysis of the differentially expressed miRNA target genes showed that they play a role in maintaining cell internal and external environment stability, gene transcription, translation and expression, biochemical metabolic processes, cell proliferation and differentiation, signal transduction and apoptosis. Target gene prediction and KEGG function enrichment analysis found that differentially expressed miRNA target gene-related signaling pathways mainly include the 'FoxO signaling pathway', 'mTOR signaling pathway', 'ErbB signaling pathway', 'insulin signaling pathway', 'focal adhesion signaling pathway', 'PI3K-Akt signaling pathway', 'MAPK signaling pathway', 'cell cycle', 'Hippo signaling pathway' and 'Wnt signaling pathway'. In the FoxO signaling pathway, FoxO transcription factor changes play an important role in cell proliferation, apoptosis, differentiation and resistance to oxidative stress and other aspects. Among them, the transcription factor FoxO1 may be closely related to tumorigenesis. Low expression of miR-181a2/miR-181b2 can promote tumor growth of cervical cancer through the PIK3R3/Akt/FoxO signaling pathway (27). miR-486-5p acts as a powerful prostate cancer driver which drives tumorigenesis by directly targeting FoxO signaling (28).

Overactivation of the mTOR signaling pathway is associated with tumorigenesis and is an important target for tumor therapy. Regulation of the mTOR signaling pathway through miRNAs and control of miRNA biogenesis through mTORs may be important for the diagnosis and treatment of different types of human cancer. Previous studies have shown that miR-126 targets the PI3K/AKT/mTOR signaling pathway, maintains the stemness of leukemia stem cells and promotes chemotherapy resistance $(29,30)$. By inhibiting hematopoietic pre-B cell leukemia transcription factor-interacting protein-mediated mTOR signaling pathway, miR-148a can reduce the growth, epithelial-to-mesenchymal transition (EMT), invasion, and metastasis of HBx-expressing hepatocarcinoma cells (31). 
Table II. Results of the KEGG pathway analysis of the differentially expressed miRNAs.

\begin{tabular}{|c|c|c|c|}
\hline Term & Sample number & Background number & P-value \\
\hline Axon guidance & 59 & 127 & 0.014083421 \\
\hline FoxO signaling pathway & 70 & 133 & 0.000780811 \\
\hline Endocytosis & 96 & 203 & 0.001494435 \\
\hline mTOR signaling pathway & 37 & 60 & 0.001804281 \\
\hline Ubiquitin mediated proteolysis & 68 & 137 & 0.002811886 \\
\hline Pancreatic cancer & 38 & 66 & 0.003796989 \\
\hline Pathways in cancer & 138 & 327 & 0.005127192 \\
\hline Chronic myeloid leukemia & 40 & 73 & 0.005717632 \\
\hline Adherens junction & 39 & 73 & 0.008543298 \\
\hline Renal cell carcinoma & 36 & 66 & 0.008872749 \\
\hline Acute myeloid leukemia & 32 & 57 & 0.009730435 \\
\hline ErbB signaling pathway & 44 & 88 & 0.012823109 \\
\hline $\begin{array}{l}\text { Arrhythmogenic right ventricular } \\
\text { cardiomyopathy (ARVC) }\end{array}$ & 38 & 74 & 0.014654654 \\
\hline Insulin signaling pathway & 64 & 141 & 0.015666822 \\
\hline Focal adhesion & 88 & 206 & 0.017299249 \\
\hline PI3K-Akt signaling pathway & 138 & 346 & 0.020570672 \\
\hline Glioma & 33 & 65 & 0.024338625 \\
\hline Fc gammaR-mediated phagocytosis & 43 & 91 & 0.026594706 \\
\hline Dopaminergic synapse & 58 & 131 & 0.02879438 \\
\hline MAPK signaling pathway & 104 & 257 & 0.030410368 \\
\hline mRNA surveillance pathway & 42 & 91 & 0.036289303 \\
\hline Rap1 signaling pathway & 87 & 213 & 0.038127362 \\
\hline SNARE interactions in vesicular transport & 20 & 36 & 0.038478648 \\
\hline Ras signaling pathway & 92 & 227 & 0.038565721 \\
\hline Non-small cell lung cancer & 28 & 56 & 0.040725456 \\
\hline Cell cycle & 54 & 124 & 0.041455661 \\
\hline Hippo signaling pathway & 65 & 154 & 0.042550777 \\
\hline Thyroid hormone signaling pathway & 52 & 119 & 0.04292765 \\
\hline Transcriptional misregulation in cancer & 74 & 179 & 0.043938096 \\
\hline Colorectal cancer & 30 & 62 & 0.046445737 \\
\hline Endometrial cancer & 26 & 52 & 0.047323945 \\
\hline Circadian rhythm & 17 & 30 & 0.047830477 \\
\hline
\end{tabular}

The sample number indicates the number of enrichment genes in the comparison of the group participating in the signaling pathway. The background number indicates how many genes in the reference genomic information of the species participate in the signaling pathway process. The former is the significantly different gene in the experimental results, and the latter is the data in the reference genome of the studied species. KEGG, Kyoto Encyclopedia of Genes and Genomes.

Studies have also shown that inhibition of miRNAs in the mTOR signaling pathway may inhibit the growth of tumor cells. In addition, miR-590-3p/MACC1 was found to inhibit the malignant biological behavior of glioblastoma stem cells by inhibiting the PI3K/AKT/mTOR pathways (32-35). There are also drugs that inhibit cell proliferation and tumor growth of esophageal adenocarcinoma both in vitro and in vivo via the AMPK $\alpha / m$ TOR signaling pathway (36).

The focal adhesion pathway is closely related to EMT, which directly or indirectly regulates EMT-related protein expression and cytoskeletal remodeling, thereby affecting the EMT process $(37,38)$. It plays a role in the formation and differentiation of bone and cartilage (39). The focal adhesion signaling pathway mediates the involvement of miR-92a in cartilage formation and chondrocyte response induced by IL-1 $\beta$ (40). In the field of cancer, its involvement in miR-301/PTEN (phosphatase and tensin homolog) promotes the progression of malignant melanoma (41).

Previous studies have shown that miR-29c-5p, miR-29b and miR-193a-3p all participate in the MAPK signaling pathway, and methylation-associated silencing of miR-193a-3p promotes ovarian cancer aggressiveness by targeting MAPK/ERK pathways (42). miR-29c-5p inhibits gallbladder carcinoma progression by directly targeting cytoplasmic polyadenylation element binding protein 4 and inhibiting the MAPK signaling pathway (43). miR-29b negatively modulates the MAPK/ERK 


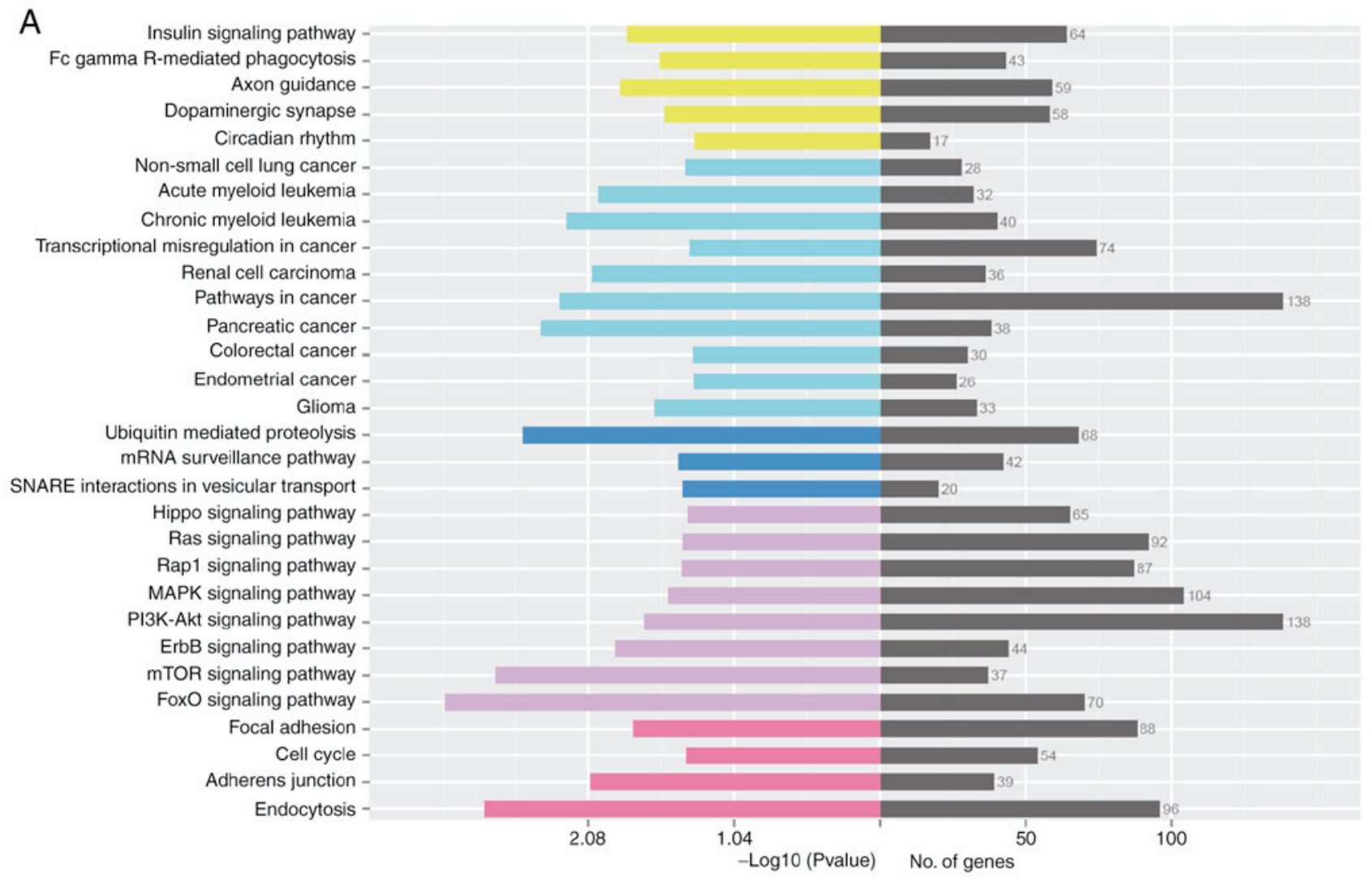

B

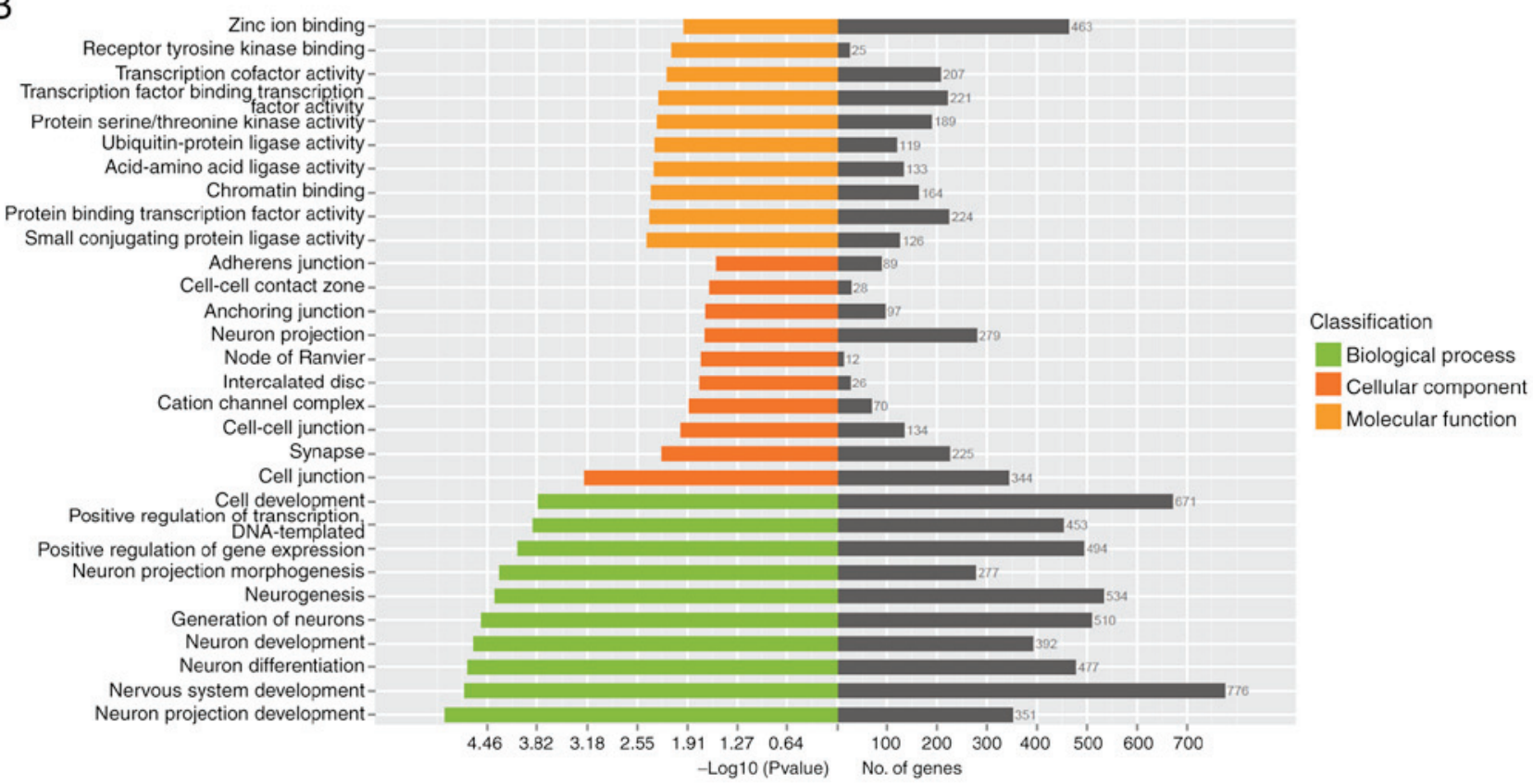

Figure 4. (A) Kyoto Encyclopedia of Genes and Genomes pathway classification of the differentially expressed miRNA target genes in the experimental and control groups. (B) Gene Ontology enrichment map of the differentially expressed miRNA target genes. miRNA, microRNA.

and PI3K/Akt signaling pathways to inhibit angiogenesis in endometrial carcinomas by targeting vascular endothelial growth factor A (44).

The Wnt signaling pathway plays a role in stem cells. miR-214 is a key regulator of Wnt signaling pathway activity and stem cell function during normal tissue homeostasis, regeneration and aging (45). The Wnt signaling pathway is an important signal transduction pathway for the differentiation of mesenchymal stem cells into cardiomyocytes, which is regulated by miR-1-2. Overexpression of miR-1-2 in bone marrow-derived stem cells (BMSCs) of mice can induce differentiation into cardiomyocytes by activating the Wnt/ $\beta$-catenin signaling pathway (46). At the same time, it also plays an important role in the invasion and treatment of tumors. By activating the $\mathrm{Wnt} / \beta$-catenin signaling pathway, miR-106b-5p promotes invasiveness of renal cell carcinoma 
and stem cell-like phenotype (47). Low levels of miR-600 are correlated with activation of the Wnt signaling pathway and poor prognosis in breast cancer) (48).

EpSCs are the key source of skin damage repair. The present study found that expression of miRNAs was significantly altered after heat loss. If various strategies can be used to activate endogenous stem cells, then it is beneficial to help the wound healing direction of patients with extensive burns (49). At the same time, there is increasing evidence that the recruitment of mesenchymal stem cells is beneficial for tissue repair after injury. It has been reported that migration of mesenchymal stem cells may contribute to tumor angiogenesis (50); however, the present study used the patient's own epidermal stem cells as this eliminates the risk factors that may cause mesenchymal stem cells to facilitate tumor angiogenesis, and have a good application prospect.

Overall, through high-throughput sequencing technology, the present study found that miRNAs in human EpSCs, following heat injury were significantly differentially expressed which may be related to the mechanism of the wound healing signaling pathway. However, the current research is still at a preliminary stage. Expression of miRNAs needs to be further modified and intervened to clarify the specific regulatory mechanisms that are involved during the wound healing process, as well as provide more information on the safe and effective application of targeted treatment of various types of wounds.

\section{Acknowledgements}

The authors would like to thank Mrs Meng-Yun Li, Mr Shang-Feng Fu and Mr Long-Xiang Tu, all from The First Affiliated Hospital of Nanchang University (Nanchang, China); Mr Ji Yan from The First Clinical Medical College, Nanchang University (Nanchang, China) and Mr Xing-Chao Liu from Beihang University (Beijing, China) for their guidance and assistance.

\section{Funding}

The present study was funded by the National Natural Science Foundation of China (grant no. 81460293).

\section{Availability of data and materials}

The datasets used during the present study are available from the corresponding author upon reasonable request.

\section{Authors' contributions}

DWL designed the present study. HTR and DWL performed the experiments, wrote the paper, and reviewed and edited the initial manuscript. Both authors read and approved the final published version of the manuscript.

\section{Ethics approval and consent to participate}

The present study was performed according to The Declaration of Helsinki and was approved by the Ethics Committee of Nanchang University (Nanchang, China). Written informed consent was provided by all the participating donors.

\section{Patient consent for publication}

Not applicable.

\section{Competing interests}

The authors declare that they have no competing interests.

\section{References}

1. Gurtner GC, Werner S, Barrandon Y and Longaker MT: Wound repair and regeneration. Nature 453: 314-321, 2008.

2. Bao P, Kodra A, Tomic-Canic M, Golinko MS, Ehrlich HP and Brem H: The role of vascular endothelial growth factor in wound healing. J Surg Res 153: 347-358, 2009.

3. Xue M, Zhao R, Lin H and Jackson C: Delivery systems of current biologicals for the treatment of chronic cutaneous wounds and severe burns. Adv Drug Deliv Rev 129: 219-241, 2018.

4. Werner S and Grose R: Regulation of wound healing by growth factors and cytokines. Physiol Rev 83: 835-870, 2003.

5. Horsburgh S, Fullard N, Roger M, Degnan A, Todryk S, Przyborski S and O'Reilly S: MicroRNAs in the skin: Role in development, homoeostasis and regeneration. Clin Sci (Lond) 131: 1923-1940, 2017.

6. Brouard $M$ and Barrandon Y: In-vivo dedifferentiation of keratinocytes to epidermal stem cells. Lancet 359: 528-529, 2002.

7. Levy V, Lindon C, Zheng Y, Harfe BD and Morgan BA: Epidermal stem cells arise from the hair follicle after wounding. FASEB J 21: 1358-1366, 2007.

8. Blanpain $C$ and Fuchs E: Epidermal stem cells of the skin. Annu Rev Cell Dev Biol 22: 339-373, 2006.

9. Beck B and Blanpain C: Mechanisms regulating epidermal stem cells. EMBO J 31: 2067-2075, 2012.

10. Grosshans H and Slack FJ: Micro-RNAs: Small is plentiful. J Cell Biol 156: 17-21, 2002.

11. Lewis CJ: Stem cell application in acute burn care and reconstruction. J Wound Care 22: 7-8, 10, 12-16, 2013.

12. Song Z, Liu D, Peng Y, Li J, Zhang Z and Ning P: Differential microRNA expression profile comparison between epidermal stem cells and differentiated keratinocytes. Mol Med Rep 11: 2285-2291, 2015.

13. Liu Y, Zhong L, Liu D, Ye H, Mao Y and Hu Y: Differential miRNA expression profiles in human keratinocytes in response to protein kinase C inhibitor. Mol Med Rep 16: 6608-6619, 2017.

14. Qu M and Nourbakhsh M: Current experimental models of burns. Discov Med 23: 95-103, 2017.

15. Banerjee J and Sen CK: microRNA and wound healing. Adv Exp Med Biol 888: 291-305, 2015.

16. Herter EK and Xu Landén N: Non-coding RNAs: New players in skin wound healing. Adv Wound Care (New Rochelle) 6: 93-107, 2017.

17. Meng Z, Zhou D, Gao Y, Zeng M and Wang W: miRNA delivery for skin wound healing. Adv Drug Deliv Rev 129: 308-318, 2018.

18. Berindan-Neagoe I, Monroig PC, Pasculli B and Calin GA: MicroRNAome genome: A treasure for cancer diagnosis and therapy. CA Cancer J Clin 64: 311-336, 2014.

19. Tang L, Chen HY, Hao NB, Tang B, Guo H, Yong X, Dong H and Yang SM: Microrna inhibitors: Natural and artificial sequestration of microrna. Cancer Lett 407: 139-147, 2017.

20. Paul CP, Good PD, Li SX, Kleihauer A, Rossi JJ and Engelke DR: Localized expression of small RNA inhibitors in human cells. Mol Ther 7: 237-247, 2003.

21. Mullard A: Oncology trials gear up for high-throughput sequencing. Nat Rev Drug Discov 11: 339-340, 2012.

22. Yang L, Zheng Z, Zhou Q, Bai X, Fan L, Yang C, Su L and Hu D: miR-155 promotes cutaneous wound healing through enhanced keratinocytes migration by MMP-2. J Mol Histol 48: 147-155, 2017.

23. Mori R, Tanaka $\mathrm{K}$ and Shimokawa I: Identification and functional analysis of inflammation-related miRNAs in skin wound repair. Dev Growth Differ 60: 306-315, 2018.

24. Fomicheva KA, Knyazev EN and Mal'tseva DV: hsa-miR-1973 MicroRNA is significantly and differentially expressed in MDA-MB-231 cells of breast adenocarcinoma and xenografts derived from the tumor. Bull Exp Biol Med 163: 660-662, 2017. 
25. Wang B, Li J, Sun M, Sun L and Zhang X: miRNA expression in breast cancer varies with lymph node metastasis and other clinicopathologic features. IUBMB Life 66: 371-377, 2014.

26. Sen CK and Ghatak S: miRNA control of tissue repair and regeneration. Am J Pathol 185: 2629-2640, 2015.

27. Mei Q, Li X, Zhang K, Wu Z, Li X, Meng Y, Guo M, Luo G, Fu $X$ and Han W: Genetic and methylation-induced loss of miR-181a2/181b2 within chr9q33.3 facilitates tumor growth of cervical cancer through the PIK3R3/Akt/FoxO signaling pathway. Clin Cancer Res 23: 575-586, 2017.

28. Yang Y, Ji C, Guo S, Su X, Zhao X, Zhang S, Liu G, Qiu X, Zhang Q, Guo $\mathrm{H}$ and Chen $\mathrm{H}$ : The miR-486-5p plays a causative role in prostate cancer through negative regulation of multiple tumor suppressor pathways. Oncotarget 8: 72835-72846, 2017.

29. Lechman ER, Gentner B, Ng SWK, Schoof EM, van Galen P, Kennedy JA, Nucera S, Ciceri F, Kaufmann KB, Takayama N, et al: miR-126 regulates distinct self-renewal outcomes in normal and malignant hematopoietic stem cells. Cancer Cell 29: 602-606, 2016.

30. Raffel S and Trumpp A: Mir-126 drives quiescence and self-renewal in leukemic stem cells. Cancer Cell 29: 133-135, 2016.

31. Xu X, Fan Z, Kang L, Han J, Jiang C, Zheng X, Zhu Z, Jiao H, Lin J, Jiang K, et al: Hepatitis B virus $X$ protein represses miRNA-148a to enhance tumorigenesis. J Clin Invest 123 630-645, 2013

32. Zhou W, Liu L, Xue Y, Zheng J, Liu X, Ma J, Li Z and Liu Y: Combination of endothelial-monocyte-activating polypeptide-II with temozolomide suppress malignant biological behaviors of human glioblastoma stem cells via miR-590-3p/MACC1 Inhibiting PI3K/AKT/mTOR signal pathway. Front Mol Neurosci 10: 68, 2017.

33. Wang Y, Zhang X, Tang W, Lin Z, Xu L, Dong R, Li Y, Li J, Zhang Z, Li X, et al: Mir-130a upregulates mTOR pathway by targeting TSC1 and is transactivated by NF- $\kappa \mathrm{B}$ in high-grade serous ovarian carcinoma. Cell Death Differ 24: 2089-2100, 2017.

34. Matter MS, Decaens T, Andersen JB and Thorgeirsson SS: Targeting the mTOR pathway in hepatocellular carcinoma: Current state and future trends. J Hepatol 60: 855-865, 2014.

35. Minna E, Romeo P, Dugo M, De Cecco L, Todoerti K, Pilotti S, Perrone F, Seregni E, Agnelli L, Neri A, Greco A and Borrello MG: miR-451a is underexpressed and targets AKT/mTOR pathway in papillary thyroid carcinoma. Oncotarget 7: 12731-12747, 2016.

36. Fujihara S, Morishita A, Ogawa K, Tadokoro T, Chiyo T, Kato K Kobara $\mathrm{H}$, Mori $\mathrm{H}$, Iwama $\mathrm{H}$ and Masaki T: The angiotensin II type 1 receptor antagonist telmisartan inhibits cell proliferation and tumor growth of esophageal adenocarcinoma via the AMPK $\alpha / m$ TOR pathway in vitro and in vivo. Oncotarget 8 : 8536-8549, 2017.

37. Wörthmüller J, Blum W, Pecze L, Salicio V and Schwaller B: Calretinin promotes invasiveness and EMT in malignant mesothelioma cells involving the activation of the FAK signaling pathway. Oncotarget 9: 36256-36272, 2018.
38. Jin $\mathrm{H}, \mathrm{He} \mathrm{Y}$, Zhao $\mathrm{P}, \mathrm{Hu} \mathrm{Y}$, Tao J, Chen J and Huang $\mathrm{Y}$ : Targeting lipid metabolism to overcome EMT-associated drug resistance via integrin $\beta 3 /$ FAK pathway and tumor-associated macrophage repolarization using legumain-activatable delivery. Theranostics 9: 265-278, 2019.

39. Song J, Ye B, Liu H, Bi R, Zhang N, Hu J and Luo E: Fak-Mapk, hippo and Wnt signalling pathway expression and regulation in distraction osteogenesis. Cell Prolif 51: e12453, 2018.

40. Hou C, Zhang Z, Zhang Z, Wu P, Zhao X, Fu M, Sheng P, Kang Y and Liao W: Presence and function of microRNA-92a in chondrogenic ATDC5 and adipose-derived mesenchymal stem cells. Mol Med Rep 12: 4877-4886, 2015.

41. Cui L, Li Y, Lv X, Li J, Wang X, Lei Z and Li X: Expression of nicroRNA-301a and its functional roles in malignant melanoma. Cell Physiol Biochem 40: 230-244, 2016.

42. Chen K, Liu MX, Mak CS, Yung MM, Leung HY, Xu D, Ngu SF, Chan KK, Yang H, Ngan HY and Chan DW: Methylation-associated silencing of miR-193a-3p promotes ovarian cancer aggressiveness by targeting GRB7 and MAPK/ERK pathways. Theranostics 8: 423-436, 2018.

43. Shu YJ, Bao RF, Jiang L, Wang Z, Wang XA, Zhang F, Liang HB, Li HF, Ye YY, Xiang SS, et al: MicroRNA-29c-5p suppresses gallbladder carcinoma progression by directly targeting CPEB4 and inhibiting the MAPK pathway. Cell Death Differ 24: 445-457, 2017.

44. Chen HX, Xu XX, Tan BZ, Zhang Z and Zhou XD: MicroRNA-29b Inhibits angiogenesis by targeting VEGFA through the MAPK/ERK and PI3K/Akt signaling pathways in endometrial carcinoma. Cell Physiol Biochem 41: 933-946, 2017.

45. Ahmed MI, Alam M, Emelianov VU, Poterlowicz K, Patel A Sharov AA, Mardaryev AN and Botchkareva NV: MicroRNA-214 controls skin and hair follicle development by modulating the activity of the Wnt pathway. J Cell Biol 207: 549-567, 2014

46. Shen X, Pan B, Zhou H, Liu L, Lv T, Zhu J, Huang X and Tian J: Differentiation of mesenchymal stem cells into cardiomyocytes is regulated by miRNA-1-2 via WNT signaling pathway. J Biomed Sci 24: 29, 2017.

47. Lu J, Wei JH, Feng ZH, Chen ZH, Wang YQ, Huang Y, Fang Y, Liang YP, Cen JJ, Pan YH, et al: miR-106b-5p promotes renal cell carcinoma aggressiveness and stem-cell-like phenotype by activating Wnt/ß-catenin signalling. Oncotarget 8: 21461-21471, 2017.

48. El Helou R, Pinna G, Cabaud O, Wicinski J, Bhajun R, Guyon L, Rioualen C, Finetti P, Gros A, Mari B, et al: miR-600 acts as a bimodal switch that regulates breast cancer stem cell fate through WNT signaling. Cell Rep 18: 2256-2268, 2017.

49. Papaccio F, Paino F, Regad T, Papaccio G, Desiderio V and Tirino V: Concise review: Cancer cells, cancer stem cells, and mesenchymal stem cells: Influence in cancer development. Stem Cells Transl Med 6: 2115-2125, 2017.

50. Mele L, Vitiello PP, Tirino V, Paino F, De Rosa A, Liccardo D, Papaccio $G$ and Desiderio V: Changing paradigms in Cranio-facial regeneration: Current and new strategies for the activation of endogenous stem cells. Front Physiol 7: 62, 2016. 